\title{
Application of Radial Basis Function Method for Solving Nonlinear Integral Equations
}

\author{
Huaiqing Zhang, Yu Chen, Chunxian Guo, and Zhihong Fu \\ The State Key Laboratory of Transmission Equipment and System Safety and Electrical New Technology, \\ Chongqing University, Chongqing 400044, China
}

Correspondence should be addressed to Huaiqing Zhang; zhanghuaiqing@cqu.edu.cn

Received 13 July 2014; Revised 28 August 2014; Accepted 30 August 2014; Published 28 October 2014

Academic Editor: Song Cen

Copyright (C) 2014 Huaiqing Zhang et al. This is an open access article distributed under the Creative Commons Attribution License, which permits unrestricted use, distribution, and reproduction in any medium, provided the original work is properly cited.

The radial basis function (RBF) method, especially the multiquadric (MQ) function, was proposed for one- and two-dimensional nonlinear integral equations. The unknown function was firstly interpolated by MQ functions and then by forming the nonlinear algebraic equations by the collocation method. Finally, the coefficients of RBFs were determined by Newton's iteration method and an approximate solution was obtained. In implementation, the Gauss quadrature formula was employed in one-dimensional and two-dimensional regular domain problems, while the quadrature background mesh technique originated in mesh-free methods was introduced for irregular situation. Due to the superior interpolation performance of MQ function, the method can acquire higher accuracy with fewer nodes, so it takes obvious advantage over the Gaussian RBF method which can be revealed from the numerical results.

\section{Introduction}

Consider the general form of nonlinear integral equation as follows:

$$
f(P)=\mu \int_{\Omega} k(P, Q) F[f(Q)] d Q+g(P) \quad P, Q \in \Omega,
$$

where the $g(P)$ and $k(P, Q)$ are the given functions and $P$ and $Q$ are coordinate variables of integral region $\Omega . F[f(Q)]$ is the nonlinear function of unknown $f(Q), \mu$-the known parameter. These types of nonlinear integral equations have arisen from various branches of applied science as solid and fluid mechanics, electrostatics, heat transfer, diffraction, scattering of waves, and so forth [1]. Analytical solutions either do not exist or are hard to find.

However, significant progress in this area has been obtained in the last 20 years. The projection, iterated projection, and Nystrom methods [2] are the commonly used approaches for the numerical solutions of nonlinear integral equations. But for nonrectangular region, the above methods need to divide the solution region into nonoverlapping triangular fragments and then discretize the integral equation based on approximation and numerical integration over the segments. Recent contributions in this area include Chebyshev polynomials $[3,4]$, wavelets method $[5,6]$, Sinccollocation method [7], hybrid of block-pulse function and Taylor series method [8], and orthogonal triangular functions [9].

RBFs were introduced in [10] and formed a primary tool for multivariate interpolation. Hardy [11] showed that multiquadrics (MQs) are related to a consistent solution of the biharmonic potential problem and thus they have a physical foundation. Buhmann and Micchelli [12] have shown that RBFs are related to prewavelets. Kansa [13] illustrated firstly the idea of using RBFs collocation method for solving partial differential equations (PDEs) and forming a class of truly mesh-free method. In the past decades, RBF based methods have been applied to many PDE applications as heat transfer, $1 \mathrm{D}$ and $2 \mathrm{D}$ nonlinear Burgers' equation, shallow water equation, electromagnetic problems, and so forth. However, the RBF method in solving integral equations was initially proposed in 2006. Golbabai applied the RBF networks for solving the linear integral equations [14], the linear integrodifferential equations [15], and the nonlinear integral 
equations [16]. Parand and Rad [17] presented the RBF collocation method for one-dimensional Volterra-FredholmHammerstein integral equations. Alipanah adopted the positive definite functions [18] and Gaussian RBF [19] for solving the integral equations. The related research about nonlinear integral equations attracted a lot of attention recently. In the author's former paper [20], the MQ was introduced in solving linear integral equations and two types of integration schemes such as the Gauss quadrature formula and regional split technique were put forward. Therefore, this paper attempted to adopt the RBF method for solving nonlinear integral equations and the quadrature background mesh technique for nonrectangular region integration was proposed.

The layout of this paper is as follows. In Section 2, the $\mathrm{RBF}$ and interpolation principle were described. In Section 3, the RBF method for solving nonlinear integral equation and the numerical integration scheme for coefficients matrix were proposed. In Section 4, numerical examples are given and analyzed. Finally, the conclusions are discussed in Section 5.

\section{Radial Basis Function and Interpolation Principle}

Radial basis function $\Phi: R+\rightarrow R$ (domain: $R^{d}$ ) is defined as the function of distance $r=\left\|x-x_{j}\right\|$. The commonly used RBF contains the thin plate splines function $(\Phi(r)=$ $\left.r^{2} \ln r\right)$, Gaussian function $\left(\Phi(r)=\exp \left(-\beta r^{2}\right)\right)$, Hardy's multiquadrics (MQs) functions, compact support RBFs, and so forth. Franke has proved that the MQ method has the superior comprehensive performance in 29 kinds of scattered data interpolation methods. So in this paper, we choose the MQ as the basis function. The widely used MQ function has the following expression:

$$
\phi(\mathbf{x})=\sqrt{\|\mathbf{x}-\mathbf{c}\|^{2}+\alpha^{2}} .
$$

In above equation, $\mathbf{c}$ means the center of basis function and $\alpha$ is the shape parameter which is generally associated with the distance between adjacent centers. So $\alpha=\beta\left\|\mathbf{c}_{i}-\mathbf{c}_{j}\right\|$, and $\beta$ is also called the shape parameter.

The principle of $\mathrm{RBF}$ interpolation is regarding the unknown function as linear combination of RBFs. So the approximation function can be obtained after calculating the coefficients. For example, having a series of known data points $\left(x_{i}, f\left(x_{i}\right)\right), i=1,2, \ldots, N$, the approximation function can be constructed by RBF as follows:

$$
\widehat{f}(x)=\sum_{j=1}^{N} \lambda_{j} \phi_{j}(x)=\sum_{j=1}^{N} \lambda_{j} \sqrt{\left(x-c_{j}\right)^{2}+\alpha^{2}} .
$$

Then, substituting the interpolation data points into the above equation, we obtain

$$
f\left(x_{i}\right)=\sum_{j=1}^{N} \lambda_{j} \phi_{j}\left(x_{i}\right)=\sum_{j=1}^{N} \lambda_{j} \sqrt{\left(x_{i}-c_{j}\right)^{2}+\alpha^{2}},
$$

with its abstract matrix form

$$
\left[\Phi_{d}\right][\lambda]=\left[\mathbf{f}_{d}\right]
$$

where $\left[\mathbf{f}_{d}\right]=\left[f\left(x_{1}\right), f\left(x_{1}\right), \ldots, f\left(x_{N}\right)\right]^{T}$ is the known data, $[\lambda]=\left[\lambda_{1}, \lambda_{2}, \ldots, \lambda_{N}\right]^{T}$ is the coefficients, and $\left[\Phi_{d}\right]$ is the coefficient matrix whose element calculation formula is $\Phi_{m n}=\sqrt{\left(x_{m}-c_{n}\right)^{2}+\alpha^{2}}$.

The MQ function is globally defined which results in a full resultant coefficient matrix. And consequently, the coefficient matrix in (5) is nonsingular and usually ill-conditioned. The accuracy of the MQ solution depends heavily on the shape parameter. In general, for a fixed number of centers $N$, smaller shape parameters produce more accurate approximations but also are associated with a poorly conditioned interpolation matrix. Also, the condition number grows with $N$, for fixed values of the shape parameter $\alpha$. The choice of this optimal value is still under intensive investigation. Therefore, solving (5) can acquire

$$
[\lambda]=\left[\Phi_{d}\right]^{-1}\left[\mathbf{f}_{d}\right]
$$

The approximation value $\widehat{f}(x)$ at any point $x$ is

$$
\widehat{f}(x)=[\boldsymbol{\Phi}(x)][\lambda]=[\boldsymbol{\Phi}(x)]\left[\boldsymbol{\Phi}_{d}\right]^{-1}\left[\mathbf{f}_{d}\right]
$$

\section{Solving the Nonlinear Integral Equations Based on the RBF Interpolation}

The basic idea of the RBF method for solving nonlinear integral equation is using a linear combination of RBFs to approximate the unknown function; thus the integral equation is transformed into the nonlinear equations with RBFs and their coefficients. Then the weight coefficients can be calculated by the optimization method. And finally, an approximate representation of the unknown function can be obtained. Specifically, the RBF method for solving nonlinear integral equation is proposed as follows.

3.1. RBF Interpolation to Approximate One-Dimensional Nonlinear Integral Equation. The general form of onedimensional nonlinear integral equation is

$$
f(x)=\mu \int_{a}^{b \mid x} k(x, t) F[f(t)] d t+g(x), \quad x \in[a, b] .
$$

Here, $b \mid x$ represents the upper limit. The constant $b$ or variable $x$ is, respectively, corresponding to Fredholm or Volterra equation. The upper limit $b$ was chosen for demonstration in the following discussion. The unknown function $f(x)$ is expressed as a combination of RBFs. Thus, the approximate formula for $(8)$ is obtained:

$$
\sum_{i=1}^{N} \lambda_{i} \phi_{i}(x)=\mu \int_{a}^{b} k(x, t) F\left[\sum_{i=1}^{N} \lambda_{i} \phi_{i}(t)\right] d t+g(x)
$$


Furthermore, for any collocation point $x_{d}$ in $[a, b]$, we can get the following algebraic equation:

$$
\begin{aligned}
& \sum_{i=1}^{N} \lambda_{i} \phi_{i}\left(x_{d}\right) \\
& \quad-\mu \int_{a}^{b} k\left(x_{d}, t\right) F\left[\sum_{i=1}^{N} \lambda_{i} \phi_{i}(t)\right] d t=g\left(x_{d}\right), \quad x_{d} \in[a, b] .
\end{aligned}
$$

When choosing $M$ collocation points, hence the nonlinear algebraic equations in matrix form are formed as follows:

$$
[\mathbf{F}-\mu \mathbf{K}]=[\mathbf{G}],
$$

where $[\mathbf{G}]=\left[g\left(x_{1}\right), g\left(x_{1}\right), \ldots, g\left(x_{M}\right)\right]^{T},[\mathbf{F}]=\left[\sum_{i=1}^{N} \lambda_{i} \phi_{i}\right.$ $\left.\left(x_{1}\right), \sum_{i=1}^{N} \lambda_{i} \phi_{i}\left(x_{2}\right), \ldots, \sum_{i=1}^{N} \lambda_{i} \phi_{i}\left(x_{M}\right)\right]^{T}$. The elements in matrix $[\mathbf{K}]$ are determined by

$$
K_{d}=\int_{a}^{b} k\left(x_{d}, t\right) F\left[\sum_{i=1}^{N} \lambda_{i} \phi_{i}(t)\right] d t=\int_{a}^{b} h(t) d t .
$$

It is the definite integral of function $h(t)$ which can be calculated by the following Gauss quadrature formula:

$$
\int_{-1}^{1} h(\xi) d \xi \approx \sum_{q=1}^{Q} W_{q} h\left(\xi_{q}\right)
$$

However, there are two issues that should be mentioned in the implementation of the above Gauss quadrature. The first one is that the coefficients $[\lambda]$ are undetermined, while the integrand $h(t)$ and vector $[\mathbf{F}]$ are the functions of $[\lambda]$. Hence, (11) cannot be solved directly just as the linear algebraic equations and the iterative approach is widely adopted in this situation. Fortunately, the coefficients $[\lambda]$ are presupposed in every iteration process, and the $h(t)$ and $[\mathbf{F}]$ can be calculated thereby. The second issue is that the Gauss quadrature is defined on the interval $[-1,1]$. Then, the integral range can be transformed with formula $t=(b+a) / 2+(b-a) \xi / 2=p(\xi)$. So the final formula is

$$
\begin{aligned}
K_{d} & =\frac{b-a}{2} \int_{-1}^{1} k\left[x_{d}, p(\xi)\right] F\left[\sum_{i=1}^{N} \lambda_{i} \phi_{i}(p(\xi))\right] d \xi \\
& \approx \frac{b-a}{2} \sum_{q=1}^{Q} W_{q} k\left[x_{d}, p\left(\xi_{q}\right)\right] F\left[\sum_{i=1}^{N} \lambda_{i} \phi_{i}\left(p\left(\xi_{q}\right)\right)\right] .
\end{aligned}
$$

3.2. RBF Interpolation to Approximate Two-Dimensional Linear Integral Equation. Two-dimensional nonlinear integral equation has the general form

$$
\begin{array}{r}
f(x, y)=\mu \int_{\Omega} k(x, y, t, s) F[f(t, s)] d t d s+g(x, y), \\
(x, y) \in \Omega .
\end{array}
$$

The corresponding RBF approximation at collocation points $\left(x_{d}, y_{d}\right)$ is

$$
\begin{aligned}
& \sum_{i=1}^{N} \lambda_{i} \phi_{i}\left(x_{d}, y_{d}\right)-\mu \int_{\Omega} k\left(x_{d}, y_{d}, t, s\right) F\left[\sum_{i=1}^{N} \lambda_{i} \phi_{i}(t, s)\right] d t d s \\
& \quad=g\left(x_{d}, y_{d}\right), \quad\left(x_{d}, y_{d}\right) \in \Omega .
\end{aligned}
$$

And in a similar way, the matrix element of nonlinear equations $[\mathbf{F}-\mu \mathbf{K}]=[\mathbf{G}]$ can be obtained for $M$ collocation points case. According to the domain type, two integration schemes as regular and irregular situation were put forward.

(1) Definite Integral Calculation for Regular Domain. When the integral domain $\Omega$ is regular in $[a, b] \times[c, d]$, using the two-dimensional Gauss quadrature formula with the transform as $t=(b+a) / 2+(b-a) \xi / 2=p(\xi)$ and $s=(d+c) / 2+(c-c) \eta / 2=q(\eta)$, we have

$$
\begin{aligned}
& K_{d}=\int_{\Omega} k\left(x_{d}, y_{d}, t, s\right) F {\left[\sum_{i=1}^{N} \lambda_{i} \phi_{i}(t, s)\right] d t d s } \\
& \approx \frac{(b-a)(d-c)}{4} \sum_{l=1}^{\mathrm{Q}_{1}} \sum_{m=1}^{\mathrm{Q}_{2}} W_{l} W_{m} k\left[x_{d}, y_{d}, p\left(\xi_{l}\right), q\left(\eta_{m}\right)\right] \\
& \times F\left[\sum_{i=1}^{N} \lambda_{i} \phi_{i}\left(p\left(\xi_{l}\right), q\left(\eta_{m}\right)\right)\right] .
\end{aligned}
$$

(2) Definite Integral Calculation for Irregular Domain. The traditional and straightforward method for irregular domain integration is the regional split technique which divides the solution region into nonoverlapping triangular fragments and then calculates the integral of subregions. Therefore, the triangulations and mesh refinement are necessary which means huge time consumption. Another kind of integration techniques such as Monte Carlo method (MCM) is also preferred for approximating multidimensional integrals when the region of integral is abnormal. However, the main drawbacks of MCM are slow convergence rate and its precision for $m_{N}$-point is of $O\left(m_{N}^{-1 / 2}\right)$.

Therefore, the quadrature background mesh technique arising from meshless methods [21, 22] was introduced in this paper which can get rid of the triangulations and mesh refinement. Firstly, construct a regular extended background domain which covers the whole solution domain and then subdivide it into regular cells. Finally, the Gauss quadrature formula can be adopted as shown in Figure 1 .

It is assumed that

$$
\begin{aligned}
K_{d} & =\int_{\Omega} k\left(x_{d}, y_{d}, t, s\right) F\left[\sum_{i=1}^{N} \lambda_{i} \phi_{i}(t, s)\right] d t d s \\
& \approx \sum_{q=1}^{Q} W_{q} k\left(x_{d}, y_{d}, t_{q}, s_{q}\right) F\left[\sum_{i=1}^{N} \lambda_{i} \phi_{i}\left(t_{q}, s_{q}\right)\right] .
\end{aligned}
$$

The organization of the program is listed in Algorithm 1. 
(1) Loop over cells of domain

(2) Loop over quadrature points in every cell

(a) if quadrature point outside solution domain, go to 2(d);

(b) if quadrature point inside solution domain, compute the integrand in (18), then multiplied by the corresponding Gauss weights, go to 2(d);

(c) if quadrature point located in solution boundary, perform the similar process as 2 (b) and then multiplied by 0.5 ;

(d) end if.

(3) End quadrature point loop

(4) End cell loop

(5) Give the matrix element $K_{d}$

Algorithm 1: Flowchart for background mesh quadrature technique.

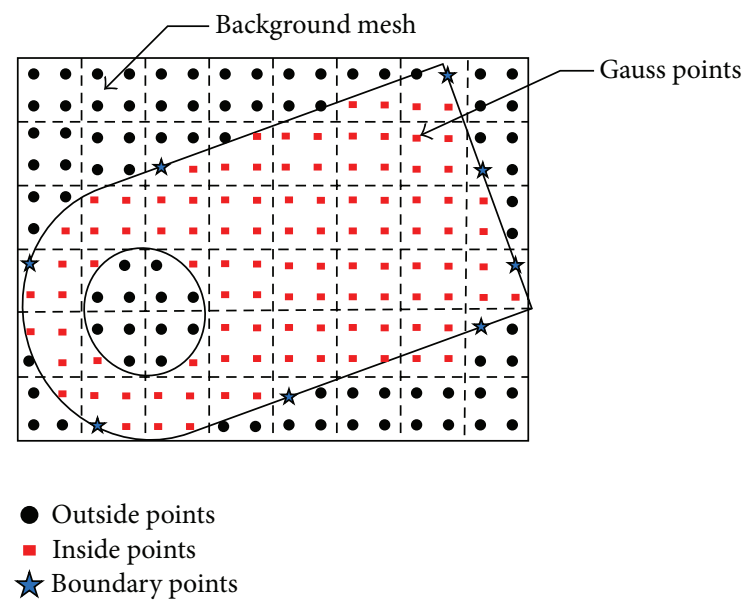

FIGURE 1: The scheme of background mesh quadrature technique.

3.3. The Implementation Procedure of RBF for Solving Nonlinear Integral Equation. In summary, the implementation procedure of RBF for solving nonlinear integral equation is listed as follows:

(1) Setting the centers $\mathbf{c}$ of RBFs and shape parameters $\alpha$ and then setting the collocation points $x_{d}$, the numbers of RBFs and collocation points are, respectively, $N$ and $M$ in general. Note that to simplify and speed up the computations, we can identify the set of centers with the set of collocation points.

(2) Substituting approximate solution based on RBF into the nonlinear integral equation, then the collocation conditions are imposed to form nonlinear algebraic equations.

(3) Matrix elements calculation schemes: the Gauss quadrature formula is adopted for one-dimensional and twodimensional regular domain problems, while, for irregular domain case, the background mesh quadrature technique was employed.

(4) Coefficient calculation: the collocation equations are the nonlinear form of RBF coefficient, and the nonlinear system of equations $[\mathbf{F}-\mu \mathbf{K}]=[\mathbf{G}]$ can be solved via Newton's iteration method to obtain unknown $[\lambda]$.

(5) RBF solution for unknown function then can be achieved as follows:

$$
f(\mathbf{x}) \approx \widehat{f}(\mathbf{x})=[\Phi(\mathbf{x})][\lambda] .
$$

\section{Numerical Experiments and Analysis}

In this section, the RBF method is applied to some numerical examples involving one-dimensional and two-dimensional integral equations on the regular and nonrectangular regions. Two types of RBF such as multiquadric (MQ) function and Gaussian function were adopted simultaneously in the following simulations. Their parameters were set the same except that the additional shape parameter was provided for MQ. In order to measure the accuracy of the method, the maximum absolute error (MAE), the maximum relative error (MRE), and the root mean squared error (RMSE) have been used, respectively. Let $f_{e}\left(x_{k}\right)$ and $f\left(x_{k}\right)$, respectively, express the exact and calculation value at the point $x_{k}$; the RMSE is defined as follows:

$$
\text { RMSE }=\sqrt{\frac{1}{m_{N}} \sum_{k=1}^{m_{N}}\left[f_{e}\left(x_{k}\right)-f\left(x_{k}\right)\right]^{2}} .
$$

Example 1. Consider the one-dimensional nonlinear integral equation [7]:

$$
\begin{array}{r}
f(x)=x \int_{0}^{1} t \sqrt{f(t)} d t+2-\frac{1}{3}(2 \sqrt{2}-1) x-x^{2}, \\
0 \leq x \leq 1 .
\end{array}
$$

The exact solution is $f(x)=2-x^{2}$.

Setting RBF centers with interval $h=1 / 10$, the collocation points are the same as the center points $N=11$. The MQ and Gaussian RBF method are implemented. For MQ, the shape parameter $\beta=14$. Then, the 10-point Gauss quadrature formula is adopted in integral calculation. And select measuring points with the spacing $h_{t}=0.01$. Table 1 lists the absolute error of the Sinc-collocation method [7], the Gaussian method, and MQ RBF method.

The RMSE, MAE, and MRE for MQ method are 7.5187E$06,1.9679 \mathrm{E}-05$, and $1.4058 \mathrm{E}-05$ but for Gaussian RBF method are $2.0951 \mathrm{E}-04,8.0920 \mathrm{E}-04$, and $4.0460 \mathrm{E}-04$, respectively. Then, the shape parameter was analyzed with interval $h=1 / 10$, and the RMSE was calculated as shown in Figure 2.

It can be seen that the MQ method can overall maintain RMSE at the level of $1 \mathrm{E}-4$ and $\beta=8,14$, and 17 are local 
TABLE 1: The absolute error of the Sinc-collocation method, Gaussian method, and MQ RBF method.

\begin{tabular}{lccc}
\hline$x_{t}$ & In [7], $N=10$ & Gaussian & MQ \\
\hline 0.1 & $4.94 E-5$ & $5.7826 e-05$ & $8.9252 e-06$ \\
0.3 & $8.55 E-5$ & $2.8593 e-04$ & $3.6018 e-06$ \\
0.5 & $1.04 E-4$ & $4.2268 e-05$ & $8.6477 e-06$ \\
0.7 & $6.78 E-5$ & $6.6820 e-05$ & $1.7214 e-07$ \\
0.9 & $1.41 E-5$ & $2.8658 e-05$ & $3.3042 e-06$ \\
MAE & $2.18 E-4$ & $2.0951 e-04$ & $7.5187 e-06$ \\
\hline
\end{tabular}

TABLE 2: Error comparison between MQ method and Gaussian method.

\begin{tabular}{|c|c|c|c|c|c|c|}
\hline \multirow{2}{*}{$h$} & \multicolumn{3}{|c|}{ MQ } & \multicolumn{3}{|c|}{ Gaussian } \\
\hline & RMSE & MAE & MRE & RMSE & MAE & MRE \\
\hline $1 / 3$ & $4.4569 e-04$ & $6.9253 e-04$ & $1.4219 e-03$ & $3.4881 e-04$ & $5.5047 e-04$ & $1.2993 e-03$ \\
\hline $1 / 7$ & $6.5120 e-06$ & $2.3456 e-05$ & $2.4413 e-05$ & $9.2277 e-03$ & $2.8376 e-02$ & $2.8376 e-02$ \\
\hline $1 / 15$ & $7.1522 e-06$ & $1.4779 e-05$ & $2.9995 e-05$ & $2.2472 e-03$ & $6.1536 e-03$ & $8.3278 e-03$ \\
\hline $1 / 31$ & $6.8676 e-06$ & $1.9591 e-05$ & $4.9179 e-05$ & $2.0452 e-03$ & $8.4702 e-03$ & $8.4702 e-03$ \\
\hline
\end{tabular}

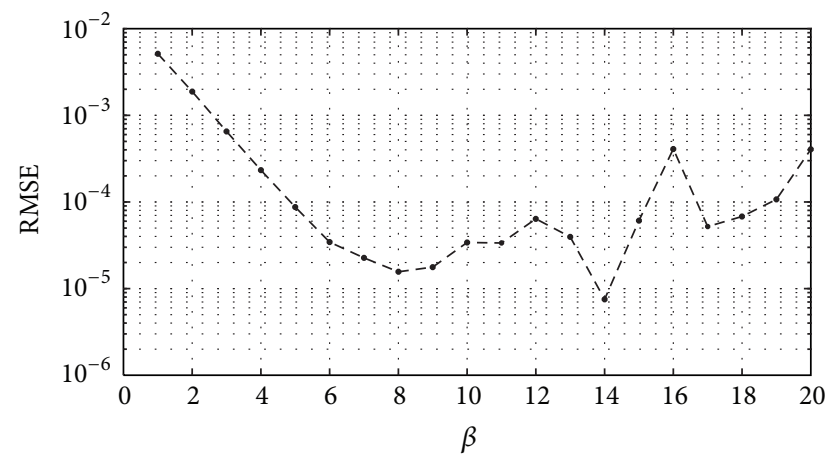

FIGURE 2: The RMSE curve versus the shape parameter $\beta$ with $h=$ $1 / 10$.

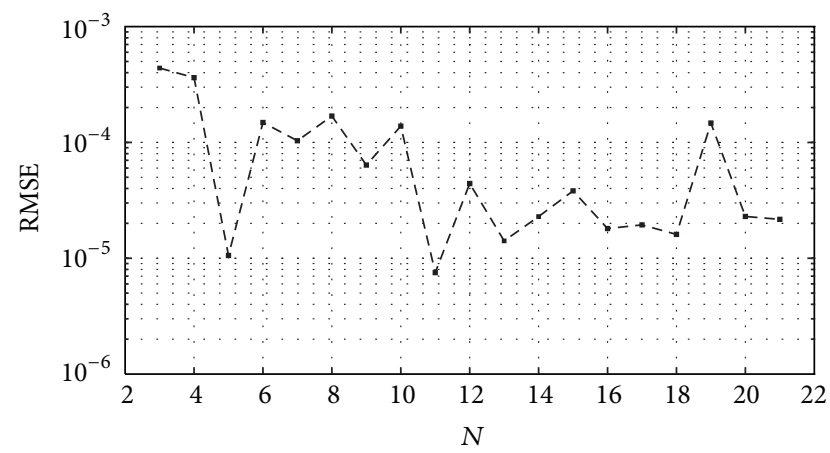

FIGURE 3: The RMSE curve versus the center nodes with $\beta=14$.

optimal shape parameters. Finally, the RBF's center nodes were analyzed in case of $\beta=14$ and were shown in Figure 3. The MQ method can achieve higher accuracy with fewer nodes; for example, the RMSE is $4.3853 \mathrm{E}$ - 04 in case of three RBF nodes. So it has obvious advantage in the aspect of calculation efficiency.

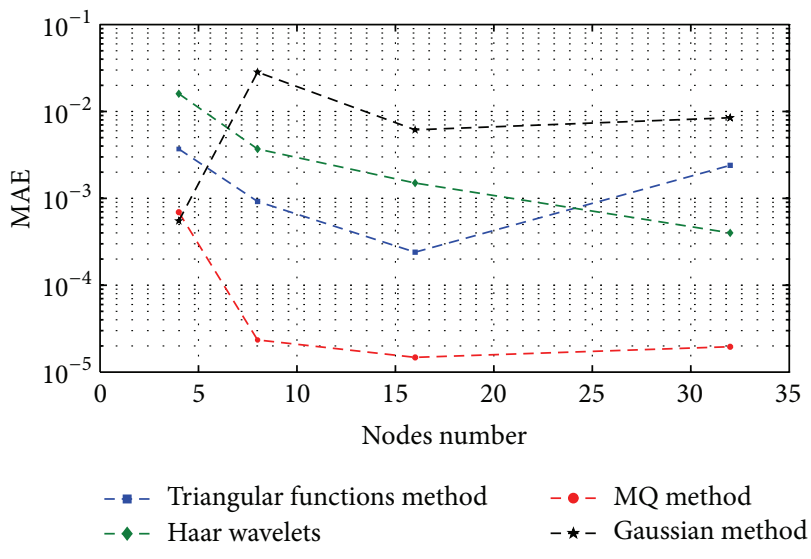

FIGURE 4: The maximum absolute error comparison among Haar, triangular, MQ, and Gaussian methods.

Example 2. Consider the following nonlinear integral equation [5]:

$$
f(x)=\frac{3}{2}-\frac{1}{2} e^{-2 x}-\int_{0}^{x}\left[f^{2}(t)+f(t)\right] d t, \quad 0 \leq x \leq 1 .
$$

The exact solution is $f(x)=\exp (-x)$.

Set the shape parameter $\beta=8$ and use 10-point Gauss quadrature formula. In order to compare with Haar Wavelet [5], successively take RBF center spacing $h=1 / 3,1 / 7,1 / 15$, and $1 / 31$; the collocation points are the same as the center points. Select testing points with the spacing $h_{t}=0.01$. Table 2 shows the calculation error with the different number of RBF nodes.

The comparisons of the maximum absolute error among Haar, triangular functions, MQ, and Gaussian methods are shown in Figure 4. 
TABLE 3: The absolute error comparison among HBT [8], Gaussian, and MQ with $h=1 / 5$.

\begin{tabular}{lccr}
\hline$x_{t}$ & In $[8], M=3$ and $N=6$ & Gaussian $(N=6)$ & MQ $(N=6)$ \\
\hline 0.0 & $0.0000 e+0$ & $2.1317 e-03$ & $2.3041 e-10$ \\
0.1 & $1.6268 e-04$ & $8.8244 e-04$ & $6.0705 e-05$ \\
0.2 & $2.4382 e-04$ & $1.5745 e-03$ & $2.2495 e-05$ \\
0.3 & $1.2754 e-04$ & $1.0464 e-03$ & $3.9094 e-05$ \\
0.4 & $2.8587 e-04$ & $1.0422 e-04$ & $7.0809 e-06$ \\
0.5 & $3.9993 e-04$ & $7.1945 e-04$ & $8.5021 e-06$ \\
0.6 & $2.2504 e-04$ & $1.1380 e-03$ & $8.3238 e-06$ \\
0.7 & $3.5946 e-04$ & $1.0789 e-03$ & $2.2438 e-05$ \\
0.8 & $1.0438 e-04$ & $6.4836 e-04$ & $1.8032 e-06$ \\
0.9 & $2.9683 e-04$ & $9.3607 e-05$ & $3.0644 e-05$ \\
\hline
\end{tabular}

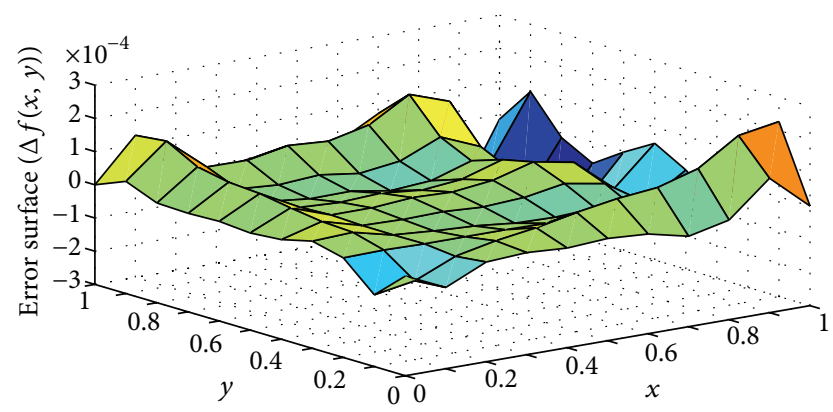

(a) MQ method

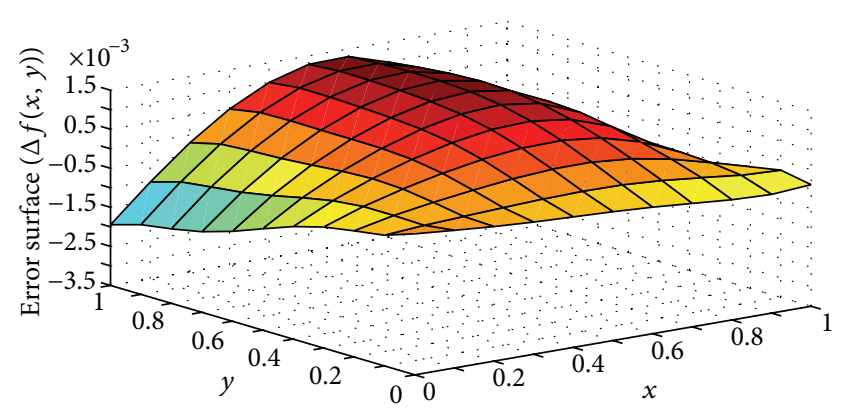

(b) Gaussian method

Figure 5: The error surfaces for MQ method and Gaussian method.

Similarly, another absolute error comparison among hybrid-block pulse functions [8], Gaussian, and MQ RBF method is listed in Table 3 with $h=1 / 5$ and $\beta=8$.

Therefore, we can conclude from Table 3 and Figure 4 that the MQ method takes obvious advantage over Gaussian, and the accuracy is improved by about 2 orders of magnitude with the raise of node number. Compared to the method in $[5,8]$, the MQ method has overall optimum precision.

Example 3. Consider the two-dimensional nonlinear integral equation [6]:

$$
\begin{gathered}
f(x, y)=g(x, y)+\int_{0}^{y} \int_{0}^{x}\left(x t^{2}+\cos (s)\right) f^{2}(t, s) d t d s \\
g(x, y)=x \sin (y)\left(1-\frac{1}{9} x^{2} \sin ^{2}(y)\right) \\
+\frac{1}{10} x^{6}\left(\frac{1}{2} \sin (2 y)-y\right), \\
0 \leq x, \quad y \leq 1 .
\end{gathered}
$$

The exact solution is $f(x, y)=x \sin (y)$.

Setting the RBF center spacing $h=0.2$, the collocation points and centers are $N=36$. The shape parameter is $\beta=5$ for MQ and the 10-point Gauss quadrature formula was also adopted in both $x$ and $y$ directions. The total testing points are 121 with equidistant $h_{t}=0.1$. The RMSE, MAE, and MRE for MQ are 6.4393E - 05, 2.3181E - 04, and 2.2911E - 03, respectively. However, the above values for Gaussian method are 9.0601E - 004, 3.3915E - 03, and 5.3004E - 03. Also comparing to the rational Haar function method in [6] whose overall accuracy was about level $1 \mathrm{E}-2$ and $1 \mathrm{E}-3$, the MQ acquires much higher accuracy than others. Figure 5 describes the error surfaces of MQ and Gaussian methods.

Example 4. Consider the two-dimensional nonlinear integral equation in irregular domain [1]:

$$
\begin{gathered}
f(x, y)-\int_{\Omega} \sin (x+y) e^{t+s} \frac{1}{f(t, s)+1} d t d s=g(x, y) \\
g(x, y)=x \cos (y)-1.0478001845 \sin (x+y),
\end{gathered}
$$

where $\Omega$ is the shark's fin domain which is shown in Figure 6 and determined by

$$
\begin{aligned}
\Omega=\{( & (x, y) \in R^{2}: 0<y<1, \\
& \left.\quad-\sqrt{2.25-2 y^{2}}+1.5<x<\sqrt{0.5-(y-0.5)^{2}}+1.5\right\} .
\end{aligned}
$$

The exact solution is $f(x, y)=x \cos (y)$. 


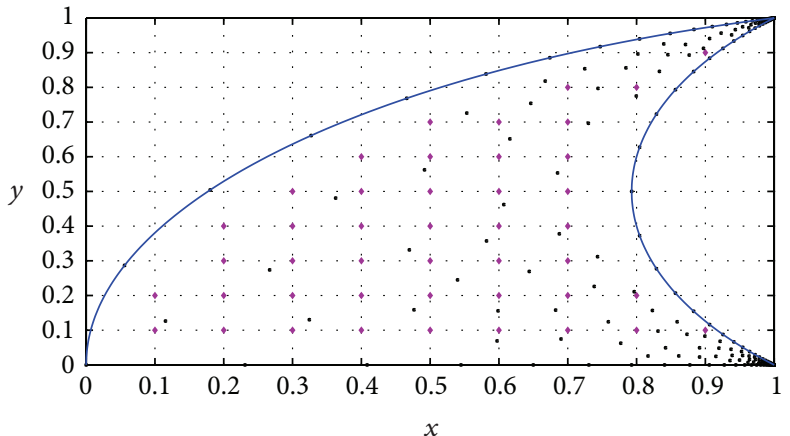

- RBF centers

- Testing points

(a) Distribution of RBF centers and testing points

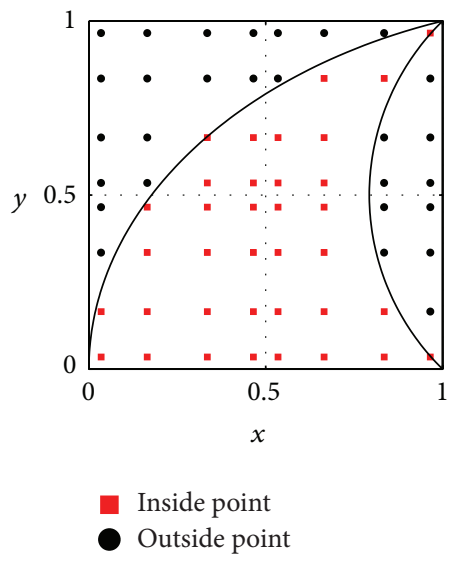

(b) Schematic diagram for background quadrature ( 4 cells and $N=4$ )

FIGURE 6: The distribution of RBF points and quadrature points in background mesh.

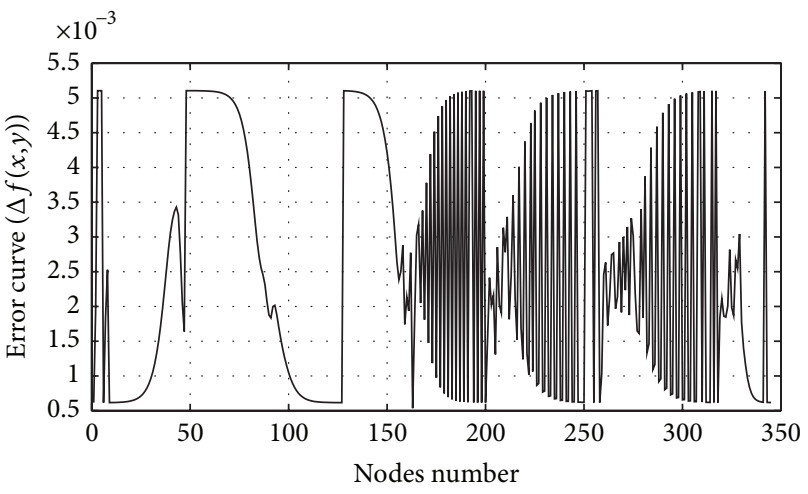

(a) MQ method

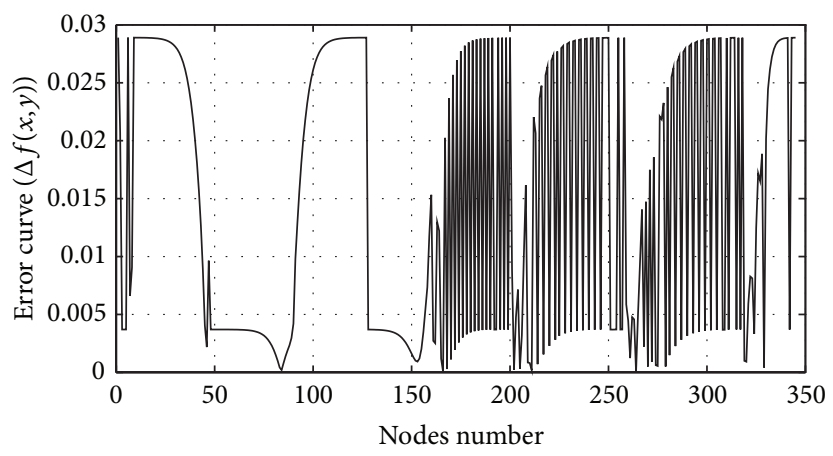

(b) Gaussian method

FIgURE 7: The absolute error curves for MQ method and Gaussian method.

Firstly, setting the RBF centers, collocation, and testing points as Figure 6, the total number of RBFs is 44 and the testing points' number is 345 . The shape parameter was $\alpha=$ $\beta h=0.8$ for MQ. The cells and Gauss points in every cell are 100 and 4 for background mesh quadrature, respectively. So there are 942 quadrature points located in the solution domain.

The results showed that the RMSE, MAE, and MRE are 3.1026E - 03, 5.1044E - 03, and 3.9887E - 02 for MQ method, while for Gaussian RBF method, the above values are $1.9129 \mathrm{E}-02,2.8901 \mathrm{E}-02$, and $2.8608 \mathrm{E}-01$, respectively. The absolute error curves of MQ and Gaussian method are shown in Figure 7. So the RBF method proposed in this paper can obtain higher accuracy.

\section{Conclusion}

In this study, we have applied an RBF, especially the MQ function, interpolation technique to solve nonlinear integral equations. The method is based on the collocation method; the Gauss quadrature formula and quadrature background mesh technique are employed for numerical integration. Compared to the Gaussian RBF and Haar wavelet methods, the MQ method can acquire better accuracy and efficiency because of its superior comprehensive interpolation performance. Numerical results showed that the MQ takes obvious advantage over Gaussian method. And in addition, the method is very convenient for solving higher dimensional integral equations because the RBF is defined as the function of distance.

\section{Conflict of Interests}

The authors declare that there is no conflict of interests regarding the publication of this paper.

\section{Acknowledgment}

This work was supported by the State Key Laboratory of Transmission Equipment and System Safety and Electrical New Technology Foundation Project 2007DA10512713210. 


\section{References}

[1] P. Assari, H. Adibi, and M. Dehghan, "A meshless method for solving nonlinear two-dimensional integral equations of the second kind on non-rectangular domains using radial basis functions with error analysis," Journal of Computational and Applied Mathematics, vol. 239, pp. 72-92, 2013.

[2] K. E. Atkinson and F. A. Potra, "Projection and iterated projection methods for nonlinear integral equations," SIAM Journal on Numerical Analysis, vol. 24, no. 6, pp. 1352-1373, 1987.

[3] Y. Liu, "Application of the Chebyshev polynomial in solving Fredholm integral equations," Mathematical and Computer Modelling, vol. 50, no. 3-4, pp. 465-469, 2009.

[4] K. Maleknejad, S. Sohrabi, and Y. Rostami, "Numerical solution of nonlinear Volterra integral equations of the second kind by using Chebyshev polynomials," Applied Mathematics and Computation, vol. 188, no. 1, pp. 123-128, 2007.

[5] I. Aziz and S. ul-Islam, "New algorithms for the numerical solution of nonlinear Fredholm and Volterra integral equations using Haar wavelets," Journal of Computational and Applied Mathematics, vol. 239, pp. 333-345, 2013.

[6] E. Babolian, S. Bazm, and P. Lima, "Numerical solution of nonlinear two-dimensional integral equations using rationalized Haar functions," Communications in Nonlinear Science and Numerical Simulation, vol. 16, no. 3, pp. 1164-1175, 2011.

[7] K. Maleknejad and K. Nedaiasl, "Application of sinc-collocation method for solving a class of nonlinear Fredholm integral equations," Computers \& Mathematics with Applications, vol. 62, no. 8, pp. 3292-3303, 2011.

[8] F. Mirzaee and A. A. Hoseini, "Numerical solution of nonlinear Volterra-Fredholm integral equations using hybrid of blockpulse functions and Taylor series," Alexandria Engineering Journal, vol. 52, no. 3, pp. 551-555, 2013.

[9] K. Maleknejad and Z. JafariBehbahani, "Applications of twodimensional triangular functions for solving nonlinear class of mixed Volterra-Fredholm integral equations," Mathematical and Computer Modelling, vol. 55, no. 5-6, pp. 1833-1844, 2012.

[10] R. L. Hardy, "Multiquadric equations of topography and other irregular surfaces," Journal of Geophysical Research, vol. 176, pp. 1905-1915, 1971.

[11] R. L. Hardy, "Theory and applications of the multiquadricbiharmonic method 20 years of discovery 1968-1988," Computers \& Mathematics with Applications, vol. 19, no. 8-9, pp. 163208, 1990.

[12] M. D. Buhmann and C. A. Micchelli, "Multiquadric interpolation improved," Computers \& Mathematics with Applications, vol. 24, no. 12, pp. 21-25, 1992.

[13] E. J. Kansa, "Multiquadrics-a scattered data approximation scheme with applications to computational fluid-dynamicsII. Solutions to parabolic, hyperbolic and elliptic partial differential equations," Computers \& Mathematics with Applications, vol. 19, no. 8-9, pp. 147-161, 1990.

[14] A. Golbabai and S. Seifollahi, "Numerical solution of the second kind integral equations using radial basis function networks," Applied Mathematics and Computation, vol. 174, no. 2, pp. 877883, 2006.

[15] A. Golbabai and S. Seifollahi, "Radial basis function networks in the numerical solution of linear integro-differential equations," Applied Mathematics and Computation, vol. 188, no. 1, pp. 427432, 2007.

[16] A. Golbabai, M. Mammadov, and S. Seifollahi, "Solving a system of nonlinear integral equations by an RBF network," Computers
\& Mathematics with Applications, vol. 57, no. 10, pp. 1651-1658, 2009.

[17] K. Parand and J. A. Rad, "Numerical solution of nonlinear Volterra-Fredholm-Hammerstein integral equations via collocation method based on radial basis functions," Applied Mathematics and Computation, vol. 218, no. 9, pp. 5292-5309, 2012.

[18] A. Alipanah and M. Dehghan, "Numerical solution of the nonlinear Fredholm integral equations by positive definite functions," Applied Mathematics and Computation, vol. 190, no. 2, pp. 1754-1761, 2007.

[19] A. Alipanah and S. Esmaeili, "Numerical solution of the two-dimensional Fredholm integral equations using Gaussian radial basis function," Journal of Computational and Applied Mathematics, vol. 235, no. 18, pp. 5342-5347, 2011.

[20] H. Q. Zhang, Y. Chen, and X. Nie, "Solving the linear integral equations based on radial basis function interpolation," Journal of Applied Mathematics, vol. 2014, Article ID 793582, 8 pages, 2014.

[21] T. Belytschko, Y. Y. Lu, and L. Gu, "Element-free Galerkin methods," International Journal for Numerical Methods in Engineering, vol. 37, no. 2, pp. 229-256, 1994.

[22] T. Belytschko, Y. Y. Lu, and L. Gu, "Crack propagation by element-free Galerkin methods," Engineering Fracture Mechanics, vol. 51, no. 2, pp. 295-315, 1995. 


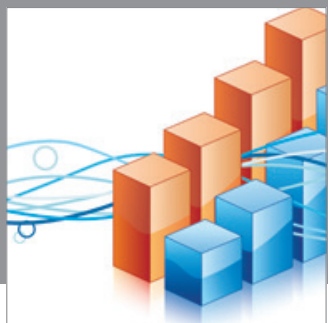

Advances in

Operations Research

mansans

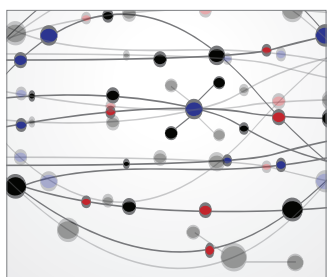

The Scientific World Journal
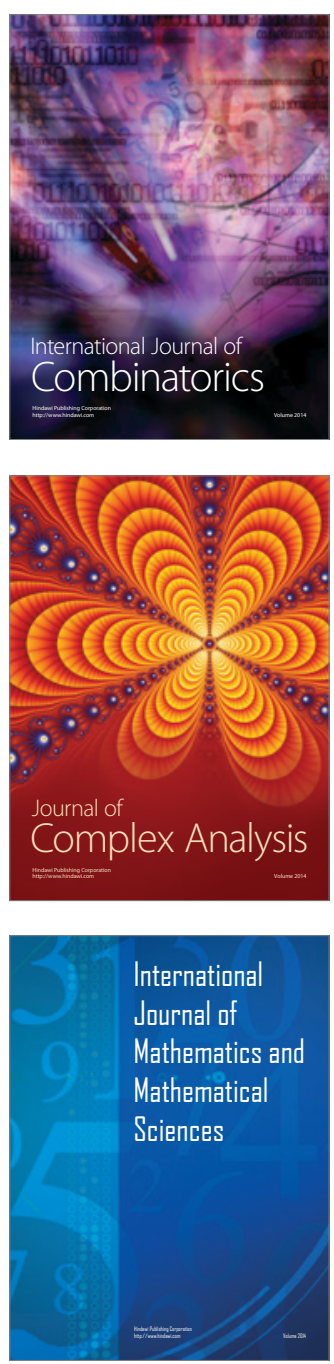
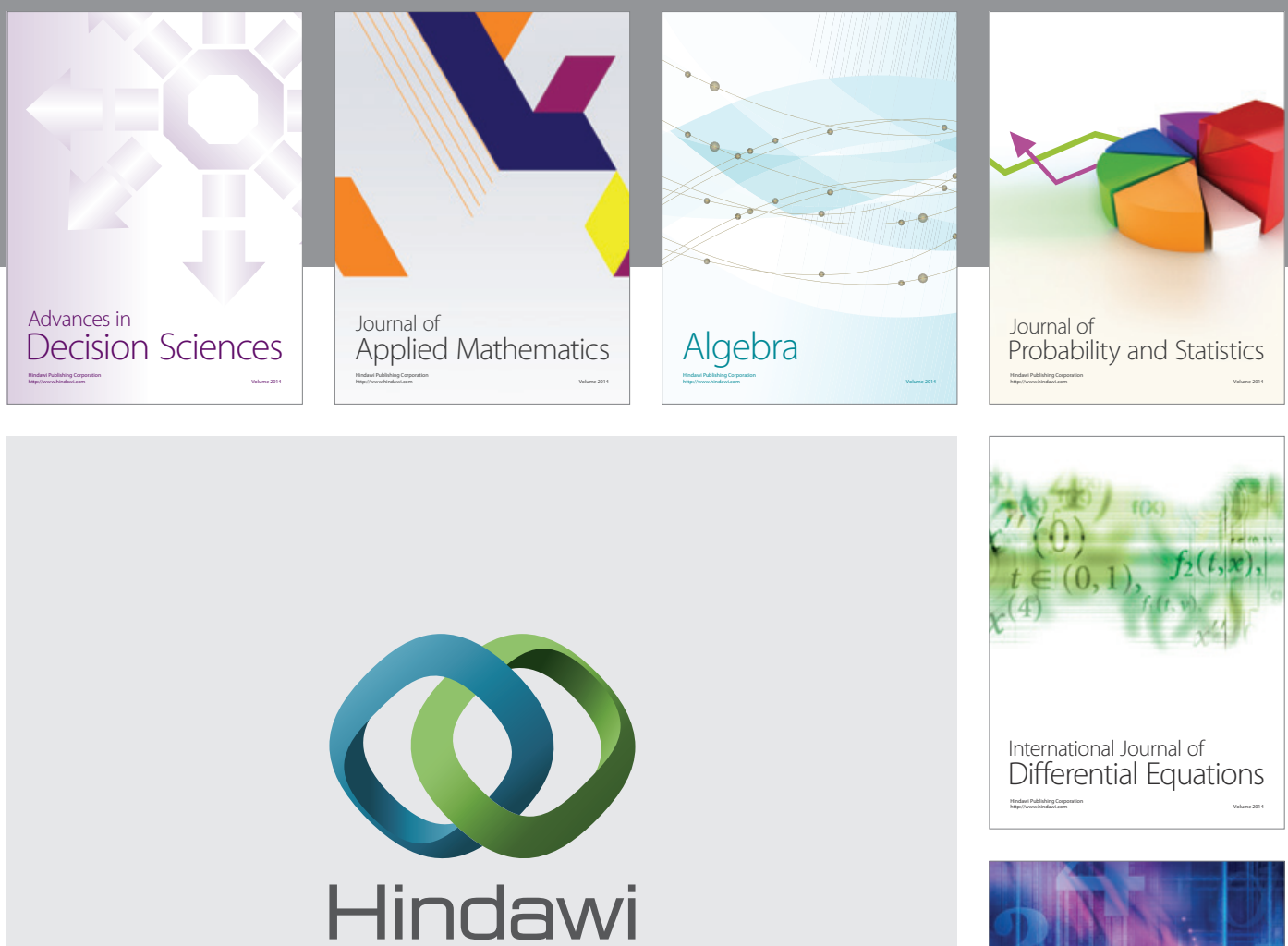

Submit your manuscripts at http://www.hindawi.com
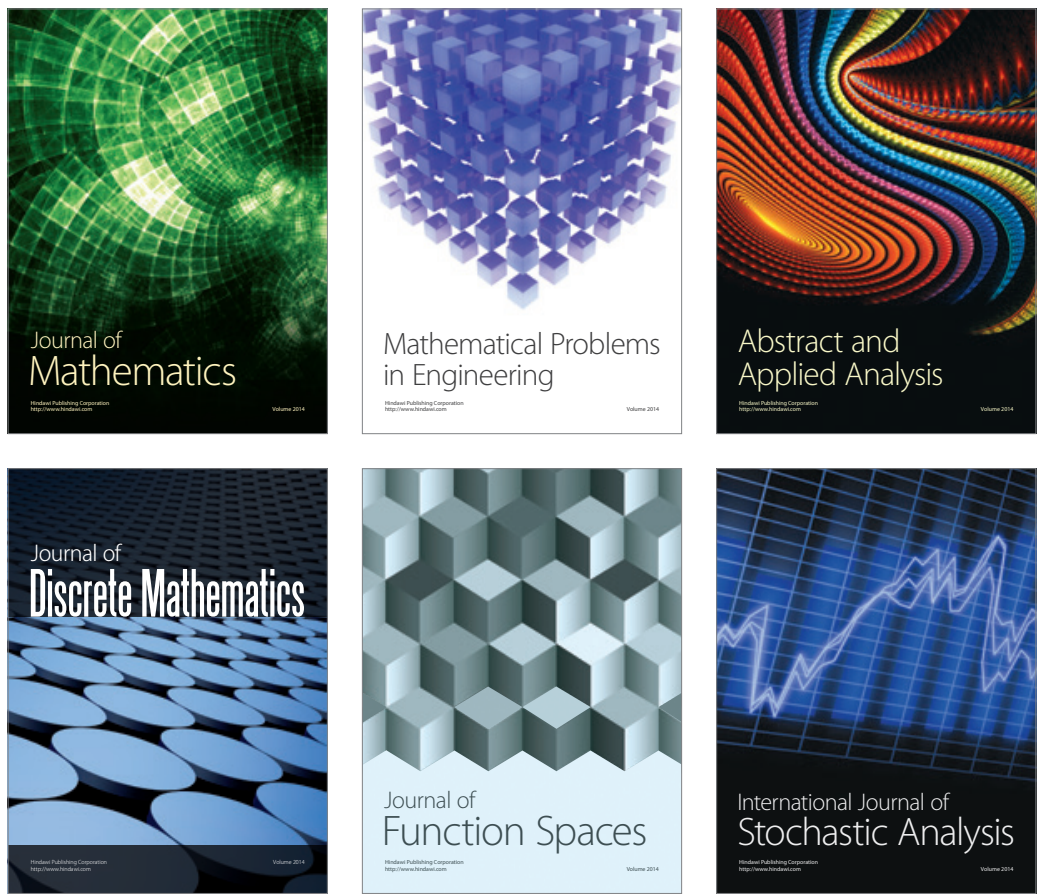

Journal of

Function Spaces

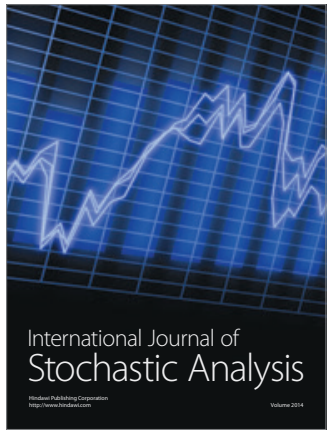

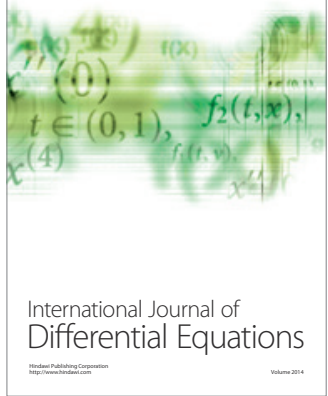
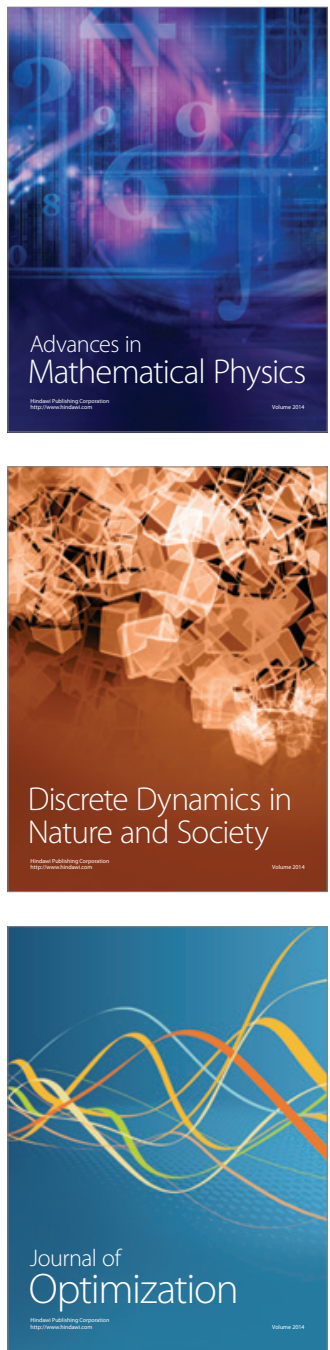